\title{
BMJ Open Metabolically healthy obesity and the risk of all-cause and cardiovascular disease mortality in a Korean population: a prospective cohort study
}

Seong-Ah Kim, ${ }^{1}$ Kyungjoon Lim, ${ }^{2}$ Jong-Koo Lee, ${ }^{3}$ Daehee Kang, ${ }^{4}$ Sangah Shin (i) ${ }^{5}$

To cite: Kim S-A, Lim K, Lee J-K, et al. Metabolically healthy obesity and the risk of all-cause and cardiovascular disease mortality in a Korean population: a prospective cohort study. BMJ Open 2021;11:e049063. doi:10.1136/ bmjopen-2021-049063

- Prepublication history for this paper is available online. To view these files, please visit the journal online (http://dx.doi. org/10.1136/bmjopen-2021049063).

Received 14 January 2021 Accepted 25 May 2021

Check for updates

(c) Author(s) (or their employer(s)) 2021. Re-use permitted under CC BY-NC. No commercial re-use. See rights and permissions. Published by BMJ.

${ }^{1}$ Department of Urban Society, Seoul Institute, Seoul, Republic of Korea

${ }^{2}$ Department of Physiology, Anatomy and Microbiology, La Trobe University, Melbourne, Victoria, Australia

${ }^{3}$ Department of Family Medicine, Seoul National University Hospital, Seoul, Republic of Korea

${ }^{4}$ Department of Preventive Medicine, College of Medicine Seoul National University, Seoul, Republic of Korea

${ }^{5}$ Department of Food and Nutrition, Chung Ang University, Anseong, Republic of Korea

Correspondence to Professor Sangah Shin; ivory8320@cau.ac.kr

\section{ABSTRACT}

Objectives This study aimed to examine the association between metabolically healthy obesity and all-cause and cardiovascular disease (CVD) mortality in a Korean population.

Design A prospective study.

Setting This study used data from the Korean Genome and Epidemiology Study.

Participants A total of 140137 participants were followed up over a median period of 9.2 years. Participants were categorised into four groups according to obesity (obese: body mass index $\geq 25 \mathrm{~kg} / \mathrm{m}^{2}$ or non-obese) and metabolic health (metabolically unhealthy: two or more metabolic abnormalities or metabolically healthy).

Primary and secondary outcome measures All-cause and CVD mortality of the participants until 31 December 2018 were ascertained by the National Health Insurance Service of beneficiary status of Korea.

Results Metabolically unhealthy non-obese participants were at elevated risk of all-cause mortality (HR, 1.12; 95\% $\mathrm{Cl}, 1.04$ to $1.21 ; \mathrm{p}=0.0019)$ and CVD mortality (HR, 1.39; $95 \% \mathrm{Cl}, 1.17$ to $1.65 ; \mathrm{p}=0.0002$ ), particularly mortality from ischaemic heart disease (IHD) (HR, 1.54; 95\% Cl, 1.10 to $2.14 ; p=0.0116$ ) compared with metabolically healthy non-obese participants. Surprisingly, metabolically healthy obese participants were at reduced risk of all-cause mortality (HR, $0.89 ; 95 \% \mathrm{Cl}, 0.81$ to $0.98 ; \mathrm{p}=0.0197$ ). Metabolically unhealthy obese participants were at elevated risk of CVD mortality (HR, $1.51 ; 95 \% \mathrm{Cl}, 1.26$ to $1.81 ; p<0.0001)$ and IHD mortality $(\mathrm{HR}, 1.88 ; 95 \% \mathrm{Cl}, 1.35$ to $2.63 ; p=0.0002$ ) compared with metabolically healthy non-obese participants

Conclusions In a Korean population, metabolically healthy obese participants had reduced risk of all-cause mortality compared with their non-obese counterparts, whereas metabolically unhealthy participants had elevated risk of CVD mortality, in particular mortality from IHD regardless of obesity.

\section{BACKGROUND}

The prevalence of obesity and its socioeconomic burden has increased worldwide. ${ }^{12} \mathrm{~A}$ considerable amount of evidence indicates that obesity is attributable to the occurrence and progression of various chronic diseases such as cardiovascular disease (CVD) and
Strengths and limitations of this study

- The present study suggests a novel approach for people with obesity to prevent and manage obesityrelated problems.

- The results of this large-scale populationrepresentative data analysis are reliable and can be generalised.

- The present study has a strength in analysing the mortality of specific type of cardiovascular diseases such as ischaemic heart diseases and cerebrovascular diseases (stroke).

- The determinants of metabolically healthy obesity, such as lifestyle factors, were not clearly elucidated in the present study.

- The dynamic concept of metabolically healthy obesity could not be considered.

ultimate death. ${ }^{3}{ }^{4}$ Moreover, obesity and impaired metabolic health have now been emerged as important risk factors for severe COVID- $19 .{ }^{5}$

Obesity is typically diagnosed based on the body mass index (BMI). ${ }^{6}$ However, among people categorised as obese based on the BMI cut-off criteria, there are individual differences in the metabolic response to obesity. ${ }^{78}$ In fact, a certain proportion of obese individuals might not be at a higher risk for metabolic complications of obesity, such as cardiovascular morbidity and mortality, than is expected for their degree of obesity. ${ }^{78}$ The subgroup of obesity in this novel concept is called metabolically healthy obesity. ${ }^{9}$

Various criteria have been used to define metabolically healthy obesity, but the common key parameter for defining 'metabolically healthy' has not been established yet. $^{7-9}$ Mainly through prospective studies in Western countries, metabolically healthy obesity has been reported to not be associated with increased risk of all-cause mortality and CVD mortality, compared with metabolically 
unhealthy status. ${ }^{10-12}$ On the contrary, there have been conflicting results that obese people are at increased risk for all-cause and CVD mortality even in the absence of metabolic abnormalities, compared with metabolically healthy normal-weight individuals. ${ }^{13-16}$ This suggests that there is no healthy pattern of obesity.

Several studies have been conducted in Asian populations. As the BMI cut-off criteria for obesity $\left(\geq 25 \mathrm{~kg} / \mathrm{m}^{2}\right)$ in some Asian countries, including Korea, are different from those in the Western countries $\left(\geq 30 \mathrm{~kg} / \mathrm{m}^{2}\right),{ }^{17}$ results of the studies performed in Western countries cannot be generalised and applied to Asian populations. In Korea, there have been two studies that examine the association between metabolic health status and obesity with risk of mortality using data from the Korean National Health Insurance Service (NHIS). ${ }^{18} 19$ Both studies found that metabolically healthy obese group had a lower all-cause mortality risk compared with the metabolically healthy non-obese group; however, there were conflicting results for CVD events and mortality between two studies. ${ }^{18} 19$ One study that analysed data of 323175 adults showed that metabolically healthy obese group had a $27 \%$ lower risk for CVD mortality when compared with their nonobese counterparts. ${ }^{18}$ The other study using data for 514866 participants reported that CVD events was higher in baseline metabolically healthy obese group than metabolically healthy non-obese group. ${ }^{19}$ Moreover, a prospective cohort study analysed data of 7588 adults from the Korean Genome and Epidemiology Study (KoGES); the metabolically healthy obese group showed a marginal increase in risk for diabetes and CVD, compared with their non-obese counterparts. ${ }^{20}$ Whether the metabolically healthy obese individuals have a lower risk of mortality, in particular CVD mortality, compared with their metabolically unhealthy counterpart is controversial. ${ }^{13-16} 1819$ Therefore, the present study aimed to examine the association between metabolically healthy obesity and allcause and CVD mortality including specific types of CVD such as ischaemic heart disease (IHD) and stroke among Korean population.

\section{METHODS}

\section{Study population}

The KoGES is a large population-based prospective cohort study initiated by the Korean government (National Research Institute of Health, Centers for Disease Control and Prevention and the Ministry of Health and Welfare) ${ }^{21}$ The KoGES includes the KoGES_Ansan and Ansung Study, the KoGES_Health Examinee (HEXA) Study and the KoGES_Multi-Rural Communities Cohort Study; all study participants were recruited from the National Health Examinee Registry. ${ }^{21}$

The KoGES_Ansan and Ansung Study is a communitybased cohort study, which recruited residents from Ansan, an industrialised region located southwest of the capital city Seoul, and from Ansung, a rural area south of Seoul. ${ }^{21}$ To recruit representative samples with statistical reliability, registered residents were randomly selected and contacted via mail, telephone or home visits. A total of 10030 participants aged 40-69 years were voluntarily enrolled between 2001 and 2002. ${ }^{21}$

The KoGES_HEXA Study consists of participants recruited from the National Health Examinee Registry, which is part of the National Health Insurance Programme. ${ }^{21} 22$ This programme provides fully paid biannual health check-ups for National Health Insurance subscribers. ${ }^{22}$ This cohort consisted of recruited volunteers aged $\geq 40$ years who visited the general hospitals in the metropolitan areas or cities in Korea. A total of 173357 participants were enrolled between 2004 and 2013. ${ }^{22}$

The KoGES_Multi-Rural Communities Cohort Study was initiated to investigate risk factors for CVD in the KoGES by setting up a community-based cohort in rural counties. ${ }^{21}$ Residents aged $\geq 40$ years were recruited from 11 rural counties. A total of 28338 participants were enrolled between 2005 and 2011. ${ }^{21}$

The study protocol for KoGES was approved by the institutional review board of the Korea Centers for Disease Control and Prevention (KCDC). Written informed consent was obtained from all participants. Also, the KCDC approved the analysis of KoGES data liked to the death certificate database.

In every cohort, participants were excluded if they could not to be linked with their death information, for reasons that included disagreement on the provision of personal information $(n=54658)$, and in cases of insufficient information for diagnosis of obesity and metabolic health $(\mathrm{n}=3984)$, such as data on BMI, blood pressure (BP), fasting blood glucose (FBG), triglyceride (TG) and high-density lipoprotein cholesterol (HDL-C). We further excluded the people diagnosed with any type of cancer including lung, stomach, liver and pancreatic cancer $(\mathrm{n}=3827)$ at baseline and people with missing values for daily energy intake $(\mathrm{n}=8970)$. Ultimately, 140137 participants were included in this study.

\section{Patient and public involvement}

No patient involved.

\section{Definition of obesity and metabolic health}

Obesity and metabolic abnormalities were determined based on the results of anthropometric measurements and biochemical analysis. Obesity was defined as BMI $\geq 25 \mathrm{~kg} / \mathrm{m}^{2}$. Participants with BMI $<25 \mathrm{~kg} / \mathrm{m}^{2}$ were defined as non-obese participants. Metabolic abnormalities were defined according to the criteria of the updated National Cholesterol Education Programme Adult Treatment Panel III, ${ }^{23}$ excepting waist circumference criterion. ${ }^{24}$ Participants who satisfied at least two of the following four criteria were categorised as metabolically unhealthy: blood TG level $\geq 150 \mathrm{mg} / \mathrm{dL}$, blood HDL-C level $<40 \mathrm{mg}$ / $\mathrm{dL}$ in men or $<50 \mathrm{mg} / \mathrm{dL}$ in women, systolic $\mathrm{BP} \geq 130 \mathrm{~mm}$ $\mathrm{Hg}$ or diastolic $\mathrm{BP} \geq 85 \mathrm{~mm} \mathrm{Hg}$ and FBG level $\geq 100 \mathrm{mg}$ / 
dL. Otherwise, patients were categorised as metabolically healthy.

Participants were categorised into four groups depending on the combination of obesity and metabolic health: metabolically healthy non-obese, metabolically unhealthy non-obese, metabolically healthy obese and metabolically unhealthy obese.

\section{Ascertainment of deaths}

Follow-up through the death certificate database of the National Statistical Office was completed. Deaths of participants until 31 December 2018 were ascertained by the NHIS of beneficiary status, in which information regarding participants' deaths was derived from the Resident Register of Korea.

Cause of death was classified according to the 10th revision of the International Classification of Disease (ICD10).$^{25}$ Cause of death was classified by ICD-10 as follows: all causes of death (A00-Z99) and CVD death (I00-I99). CVD death was further classified into death due to IHD (I20-I25) and cerebrovascular disease (I60-I69).

\section{Assessment of other variables}

Sociodemographic variables, including age, sex and household income level, and health-related lifestyle variables, such as alcohol consumption, current smoking status and physical activity, were acquired through selfadministered questionnaires. Household income level was classified into four categories: less than 1000000 won/ month, 1000 000-1 999999 won/month, 2000 000-2 999999 won/month or more than 3000000 won/month. Alcohol consumption was classified into two categories: 'current drinker' (drank alcohol at the time of survey) or 'non-drinker' (never drank alcohol or abstained from alcohol). Current smoking status was classified into three categories: 'current smoker' (smoked cigarettes at the time of survey), 'past smoker' (abstained from cigarette smoking) or 'never smoker' (never smoked cigarettes). Physical activity was classified into two categories: 'active' (exercised regularly enough to sweat one's body) or 'inactive'. Energy intake of the participants was obtained from a validated semiquantitative food frequency questionnaire at baseline.

\section{Statistical analysis}

For each participant, person-years of follow-up were calculated from baseline to 31 December 2018 or until the time of death in the case of deceased participants. We used a $\chi^{2}$ test and generalised linear model analysis for categorical and continuous variables, respectively, and differences in general characteristics at baseline with respect to the categories of obesity and metabolic health were examined. After adjusting for potential confounders, the Cox proportional hazards regression analysis was performed to estimate the HR and 95\% CI of all-cause and CVD mortality according to the categories of obesity and metabolic health. Potential confounders included age (continuous), BMI (continuous), household income level, alcohol consumption, current smoking status, physical activity and energy intake (continuous). Subgroup analysis was conducted by sex. All statistical analyses were performed using SAS software, V.9.4, and a two-sided p value $<0.05$ was considered statistically significant.

\section{RESULTS}

General characteristics of study participants at baseline are displayed in table 1 . The metabolically healthy participants were less likely to be current smokers and more likely to be younger and physically active and have a higher household income than their metabolically unhealthy counterparts (all $\mathrm{p}<0.0001$ ).

Table 2 shows the HR for all-cause and CVD mortality according to the categories of obesity and metabolic health. After a median follow-up of 9.2 years, there were 4471 all-cause deaths and 835 CVD deaths (235 IHD deaths and 333 cerebrovascular disease deaths). Compared with their healthy counterparts, metabolically unhealthy non-obese participants were at elevated risk of all-cause mortality (death rate, 47.7 vs 25.5 deaths per 1000 persons; HR, $1.12 ; 95 \%$ CI, 1.04 to $1.21 ; p=0.0019$ ) and CVD mortality (death rate, 9.8 vs 4.0 deaths per 1000 persons; HR, 1.39; 95\% CI, 1.17 to 1.65 ; $\mathrm{p}=0.0002$ ), although metabolically healthy obese participants were at reduced risk for all-cause mortality (death rate, 25.3 vs 25.5 deaths per 1000 persons; HR, 0.89; 95\% CI, 0.81 to $0.98 ; \mathrm{p}=0.0197$ ). Metabolically unhealthy obese participants were at elevated risk of CVD mortality compared with metabolically healthy non-obese participants (death rate, 9.0 vs 4.0 deaths per 1000 persons; HR, 1.51 ; $95 \%$ CI, 1.26 to $1.81 ; \mathrm{p}<0.0001)$.

As a result of subgroup analysis by sex, metabolically unhealthy non-obese participants were at elevated risk of all-cause mortality and CVD mortality compared with their healthy counterparts in men (all-cause mortality: death rate, 74.8 vs 54.5 deaths per 1000 persons; HR, 1.16; $95 \%$ CI, 1.06 to $1.27 ; \mathrm{p}=0.0015$; CVD mortality: death rate, 13.0 vs 8.2 deaths per 1000 persons; HR, 1.33; 95\% CI, 1.06 to $1.67 ; \mathrm{p}=0.0149$ ), and metabolically healthy obese participants were at reduced risk of all-cause mortality compared with their non-obese counterparts in men (death rate, 38.6 vs 54.5 deaths per 1000 persons; HR, 0.88 ; $95 \%$ CI, 0.78 to 0.99 ; $\mathrm{p}=0.0444$ ). In men, metabolically unhealthy obese participants were at elevated risk of CVD mortality compared with metabolically healthy nonobese participants (death rate, 11.2 vs 8.2 deaths per 1000 persons; HR, 1.53 ; 95\% CI, 1.21 to $1.94 ; \mathrm{p}=0.0003$ ).

Table 3 displays the HR for specific cause of CVD mortality according to the categories of obesity and metabolic health. Regarding the IHD, metabolically unhealthy non-obese participants were at elevated risk of IHD mortality compared with their healthy counterparts (death rate, 2.7 vs 1.0 deaths per 1000 persons; HR, 1.54; $95 \%$ CI, 1.10 to $2.14 ; \mathrm{p}=0.0116$ ). Metabolically unhealthy obese participants were also at elevated risk of IHD mortality compared with metabolically healthy 
Table 1 General characteristic of study participants at baseline according to the categories of obesity and metabolic health

\begin{tabular}{|c|c|c|c|c|c|}
\hline & $\begin{array}{l}\text { Metabolically } \\
\text { healthy non-obese }\end{array}$ & $\begin{array}{l}\text { Metabolically } \\
\text { unhealthy non-obese }\end{array}$ & $\begin{array}{l}\text { Metabolically } \\
\text { healthy obese }\end{array}$ & $\begin{array}{l}\text { Metabolically } \\
\text { unhealthy obese }\end{array}$ & P value* \\
\hline $\mathrm{N}$ & 66667 & 26094 & 22706 & 24670 & \\
\hline Sex=man, $n(\%)$ & $19584(29.4)$ & $10508(40.3)$ & $8777(38.7)$ & $11385(46.2)$ & $<0.0001$ \\
\hline Age (years) & $52.1 \pm 8.5$ & $56.3 \pm 8.7$ & $53.8 \pm 8.4$ & $55.5 \pm 8.6$ & $<0.0001$ \\
\hline \multicolumn{6}{|c|}{ Household income level, $\mathrm{n}(\%) \dagger$} \\
\hline$<1000000$ won & $5893(8.8)$ & 3587 (13.8) & 2601 (11.5) & $3402(13.8)$ & $<0.0001$ \\
\hline 1000 000-1999 999 & $10970(16.5)$ & $4947(19.0)$ & 3930 (17.3) & $4431(18.0)$ & \\
\hline 2000 000-2 999999 & $25296(37.9)$ & $8490(32.5)$ & 8059 (35.5) & $8028(32.5)$ & \\
\hline$\geq 3000000$ & $16254(24.4)$ & $4164(16.0)$ & $4891(21.5)$ & $4292(17.4)$ & \\
\hline \multicolumn{6}{|l|}{ Alcohol consumption† } \\
\hline Non-drinker & $36621(54.9)$ & $14314(54.9)$ & $11884(52.3)$ & $12840(52.1)$ & $<0.0001$ \\
\hline Current drinker & $29827(44.7)$ & $11701(44.8)$ & $10754(47.4)$ & $11772(47.7)$ & \\
\hline \multicolumn{6}{|l|}{ Current smoking status $\dagger$} \\
\hline Never smoker & $50973(76.5)$ & $17522(67.2)$ & $15967(70.3)$ & $15684(63.6)$ & $<0.0001$ \\
\hline Past smoker & 8035 (12.1) & $4322(16.6)$ & $4034(17.8)$ & $4951(20.1)$ & \\
\hline Current smoker & $7463(11.2)$ & $4187(16.1)$ & 2636 (11.6) & 3969 (16.1) & \\
\hline \multicolumn{6}{|l|}{ Physical activity $\dagger$} \\
\hline Active & $34040(51.1)$ & $12534(48.0)$ & $11620(51.2)$ & $11603(47.0)$ & $<0.0001$ \\
\hline Inactive & $32490(48.7)$ & $13508(51.8)$ & $11033(48.6)$ & $13018(52.8)$ & \\
\hline $\operatorname{BMI}\left(\mathrm{kg} / \mathrm{m}^{2}\right)$ & $22.1 \pm 1.8$ & $22.9 \pm 1.5$ & $26.9 \pm 1.8$ & $27.4 \pm 2.1$ & $<0.0001$ \\
\hline Waist circumference $(\mathrm{cm})$ & $76.5 \pm 6.9$ & $80.4 \pm 6.5$ & $87.6 \pm 6.7$ & $89.9 \pm 6.9$ & $<0.0001$ \\
\hline Hip circumference (cm) & $91.7 \pm 4.7$ & $92.2 \pm 4.7$ & $98.7 \pm 5.0$ & $99.0 \pm 5.4$ & $<0.0001$ \\
\hline \multicolumn{6}{|l|}{ Blood pressure $(\mathrm{mm} \mathrm{Hg})$} \\
\hline Systolic & $116.8 \pm 13.3$ & $129.6 \pm 15.3$ & $121.8 \pm 13.4$ & $132.2 \pm 14.9$ & $<0.0001$ \\
\hline Diastolic & $72.9 \pm 9.1$ & $79.8 \pm 9.9$ & $76.1 \pm 9.1$ & $81.9 \pm 9.8$ & $<0.0001$ \\
\hline $\begin{array}{l}\text { Fasting blood glucose } \\
\text { (mg/dL) }\end{array}$ & $89.6 \pm 13.7$ & $104.2 \pm 27.9$ & $92.0 \pm 15.5$ & $106.4 \pm 27.3$ & $<0.0001$ \\
\hline Triglycerides (mg/dL) & $91.3 \pm 43.8$ & $182.2 \pm 112.1$ & $106.9 \pm 50.8$ & $198.5 \pm 118.9$ & $<0.0001$ \\
\hline HDL cholesterol (mg/dL) & $57.9 \pm 12.6$ & $45.7 \pm 11.0$ & $53.9 \pm 11.1$ & $44.2 \pm 10.0$ & $<0.0001$ \\
\hline
\end{tabular}

Data were shown as mean (SD) except where indicated

${ }^{*} P$ values were calculated by generalised linear model for continuous variables and $\chi^{2}$ test for categorical variables.

†Number of missing values was 20 902, 424, 394 and 291 for household income level, alcohol consumption, current smoking status and physical activity, respectively.

BMl, body mass index; HDL, high-density lipoprotein.

non-obese participants (death rate, 2.8 vs 1.0 deaths per 1000 persons; HR, 1.88 ; $95 \%$ CI, 1.35 to 2.63 ; $\mathrm{p}=0.0002$ ).

No significant association

was observed in respect to cerebrovascular disease such as stroke.

\section{DISCUSSION}

This prospective cohort study, conducted on a large population sample from Korea, showed that metabolically healthy obesity was associated with lower risk of all-cause mortality compared with metabolically healthy non-obesity after 9.2 years of follow-up, whereas metabolically unhealthy status was associated with increased risk of
CVD mortality, in particular death due to IHD, regardless of obesity.

In the present study, metabolically healthy obesity was associated with reduced risk of all-cause mortality compared with metabolically healthy non-obesity, suggesting that a phenotype of obesity truly exists. However, several meta-analyses of prospective studies that focused on the associations between metabolic health and obesity and the risk of all-cause and CVD mortality or events in Western countries, predominantly targeting a Caucasian population, reported that metabolically healthy obesity was associated with elevated risk of allcause and cardiovascular events. This suggests that obesity 


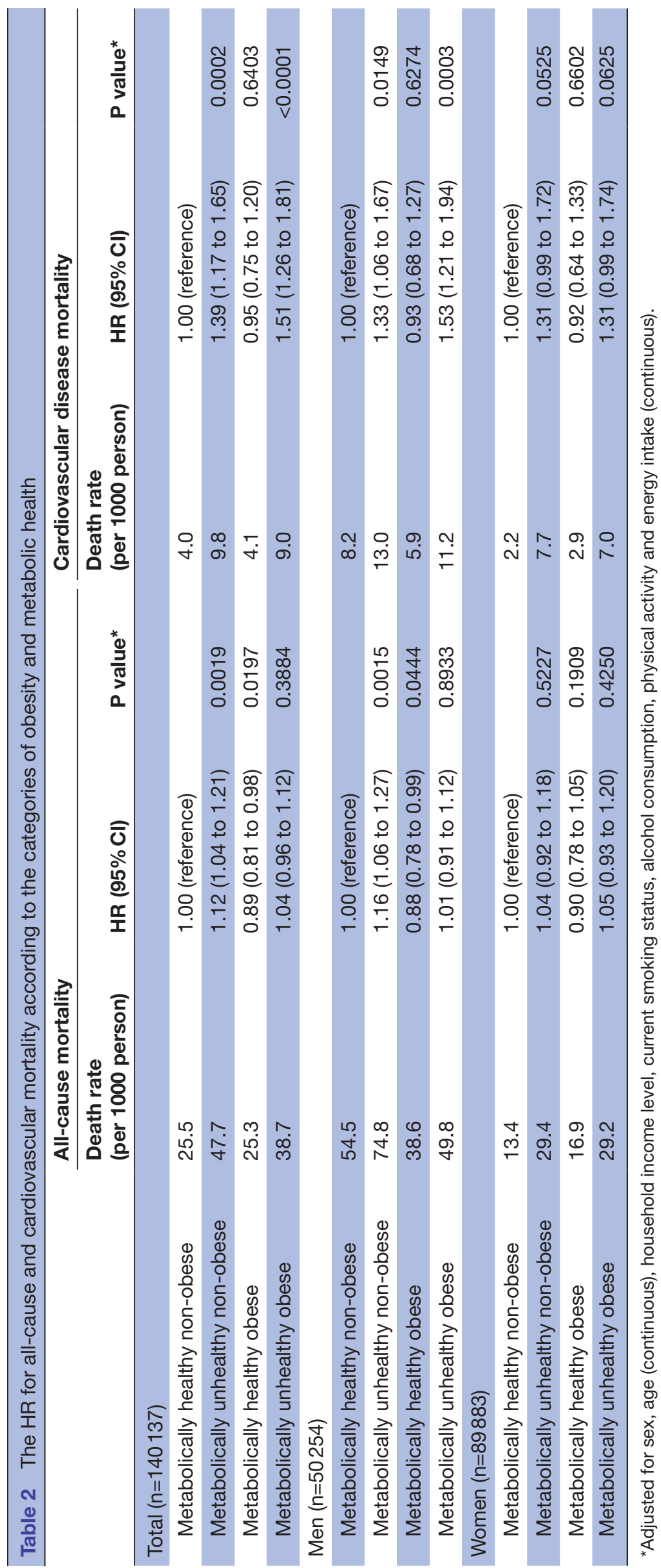




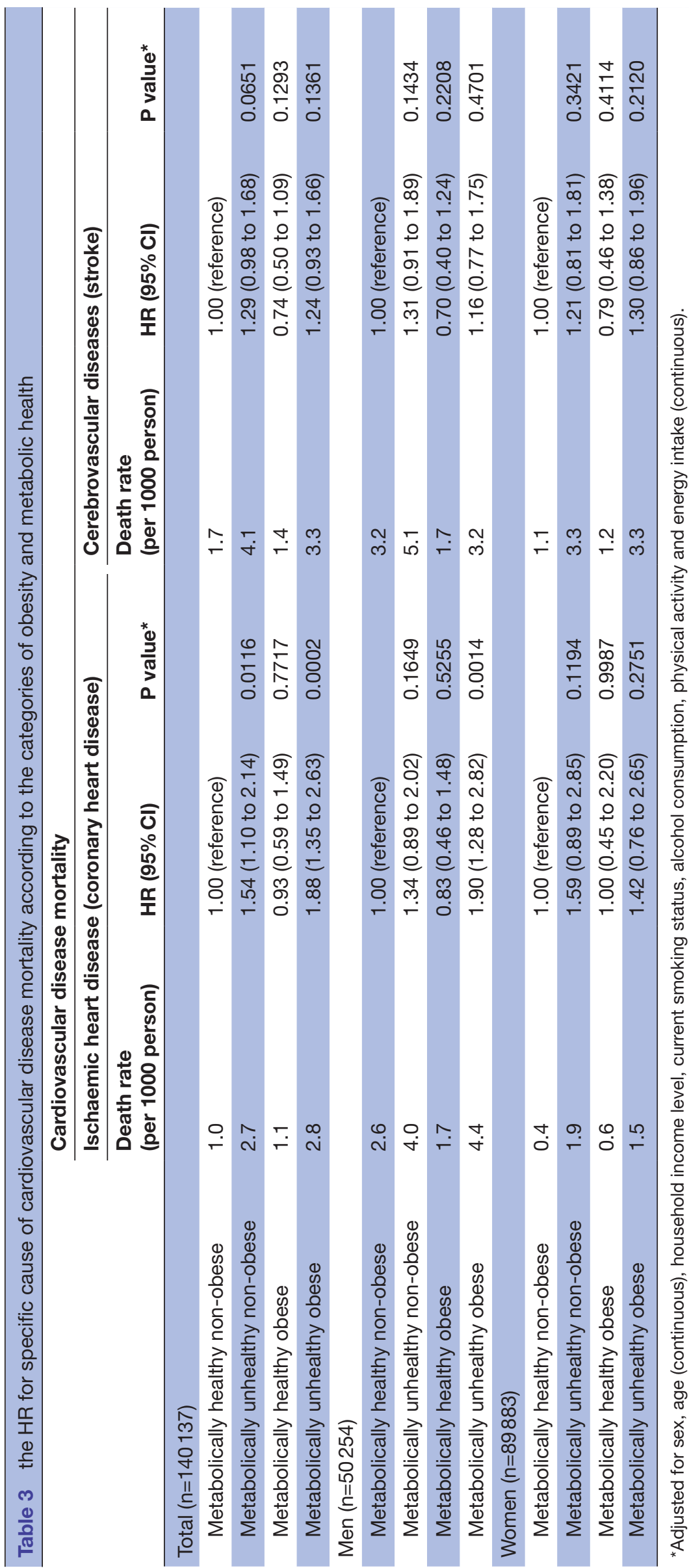

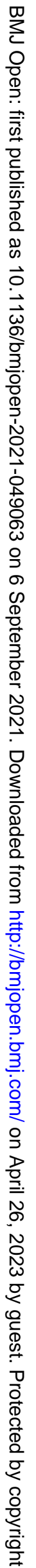


is positively associated with all-cause and CVD mortality or events, independent of metabolic health. ${ }^{14-16}$ This inconsistency of results might be due to differences in the populations and the diagnostic criteria for obesity. For Western populations, BMI cut-off criteria for obesity are $\geq 30 \mathrm{~kg} /$ $\mathrm{m}^{2}$, whereas that of some Asian countries, including Korea, is $25 \mathrm{~kg} / \mathrm{m}^{2} .{ }^{17}$ There is a possibility that within the range of $25-30 \mathrm{~kg} / \mathrm{m}^{2}$ of BMI, the point of the lowest risk for mortality may exist, which is diagnosed as obese by Asian standards but as non-obese by Western standards. It aligns with a typical J-curved or U-curved relationship between BMI and mortality. ${ }^{26}$ In a meta-analysis on the association between all-cause mortality and BMI status, BMI of $25-30 \mathrm{~kg} / \mathrm{m}^{2}$ was associated with significantly lower all-cause mortality compared with normal weight (BMI of 18.5 to $<25 \mathrm{~kg} / \mathrm{m}^{2}$ ). ${ }^{27}$ Another explanation for inconsistency in study findings is that metabolically healthy obesity is a transient state, not a permanent one. Previous studies have suggested that metabolically healthy obesity is a dynamic concept that should be considered over time. ${ }^{28}{ }^{29}$ If measured prospectively, a person who was metabolically healthy at baseline could become metabolically unhealthy before death. Previous Korean study supported that in the metabolically healthy obese people, evolving to a metabolically unhealthy status and losing weight simultaneously are associated with increased allcause mortality. ${ }^{18}$

Underlying mechanisms for the protective effects of metabolically healthy obesity on all-cause mortality can be associated with low-grade inflammation, favourable lifestyle factors and higher fat-free mass, which relates to the prevention of sarcopenia, in metabolically healthy individuals than in their metabolically unhealthy counterparts. First, previous studies found that the metabolically healthy obesity phenotype is associated with low-grade inflammatory markers such as $\mathrm{C}$ reactive protein, tumour necrosis factor- $\alpha$ and interleukin 6 , supporting a more beneficial inflammatory state compared with the metabolically unhealthy phenotype. ${ }^{30}{ }^{31}$ Second, metabolically healthy obese individuals might have healthier lifestyle compared with the metabolically unhealthy individuals. ${ }^{31}{ }^{32}$ In the present study, participants with metabolically healthy obesity were less likely to be current smokers and more likely to be physically active than metabolically unhealthy obese people. Lastly, assuming that death occurs predominantly in older people, it can be inferred that moderate to slightly higher level of BMI in old age is an indicator that reflects fat-free mass for preventing sarcopenia rather than increased body fat mass. Sarcopenia, defined as the loss of muscle mass plus low muscle strength or low physical performance, is itself an independent predictor of death in the elderly. ${ }^{33}$

In the present study, both metabolically unhealthy obese participants and metabolically unhealthy nonobese participants were at elevated risk of CVD mortality. This indicates that even for non-obese participants, metabolic abnormalities can lead to cardiovascular events and death. Specifically for a Korean population, our findings suggest that metabolic health, rather than obesity, has a greater influence on CVD mortality. The mechanism of the unfavourable association between metabolic unhealthy conditions and CVD death could be explained by a low gluteofemoral fat mass. ${ }^{34} \mathrm{Cumu-}$ lative evidence has supported that a lower gluteofemoral and leg fat mass is strongly associated with an increased cardiometabolic risk, which has been found to be the strongest predictor of a metabolically unhealthy condition, particularly in non-obese people. ${ }^{35}$ However, in the present study, gluteofemoral fat mass measured by the hip circumference ${ }^{34}$ of metabolically unhealthy participants was significantly higher than those of their healthy counterparts (table 1). There was a possibility that the higher proportion of men, who have typically higher hip circumferences compared with women, ${ }^{36}$ in metabolically unhealthy group confounded the results.

The results of subanalysis for two most common types of CVD, IHD and cerebrovascular diseases, represented by stroke, show that metabolically unhealthy participants were at elevated risk of mortality from IHD, not stroke. In a previous Korean study, people with the metabolic syndrome had $48 \%, 64 \%$ and $39 \%$ greater risks of CVD, IHD and stroke, respectively, than those without the metabolic syndrome. ${ }^{37}$ However, after adjusting for potential confounders, the increased risks for CVD and IHD remained significant. ${ }^{37}$ Although metabolically unhealthy condition has been reported to be important risk factors for both IHD and stroke, ${ }^{38-40}$ in Korean population, it seems to be more pronounced for IHD than stroke.

The results of the present study suggest a novel approach for people with obesity to prevent and manage obesityrelated problems. To date, the traditional and theoretical approach for obese persons to prevent and manage CVD is primarily losing weight, but losing a large amount of weight, in particular solely body fat mass, is not an easily achievable goal. Therefore, a practical and more sustainable alternative is to motivate people with obesity to adopt a healthy lifestyle, so that they can transition from a metabolically unhealthy status to a metabolically healthy status. Also, for healthcare professionals or medical experts, our findings provide support for prioritising the treatment of obesity in order to reduce cardiovascular risk.

This study has several limitations. First, some important risk factors for death, such as morbidity of some diseases including acute myocardial infarction, advanced heart failure and advanced liver and kidney diseases at baseline, were not included in the statistical model due to an unavailability of data. Therefore, the results should be interpreted with caution. Second, the determinants of metabolically healthy obesity, such as lifestyle factors, which can be distinguished from metabolically unhealthy counterparts, were not clearly elucidated in the present study. Third, we could not take into account the dynamic concept of metabolically healthy obesity. Because the intervals and frequency of follow-up examination were different for each cohort, participants' status of obesity and metabolic health was diagnosed only at baseline. 
In Korea, there have been a few studies to investigate the association between obesity and metabolic health status with mortality. ${ }^{18194142}$ The results of this large-scale population-representative data analysis are reliable and can be generalised. It also contributes to the growing evidence for an association between metabolically healthy obesity and mortality, since this study has a strength in analysing the mortality of specific type of CVDs such as IHDs and cerebrovascular diseases.

\section{CONCLUSION}

This large-scale prospective study among a Korean population found that metabolically healthy obese participants had lower risk of all-cause mortality than metabolically healthy non-obese people, whereas metabolically unhealthy participants had elevated risk of CVD mortality, particularly mortality from IHDs regardless of obesity status. Future research is needed to elucidate determinants of metabolically healthy obesity, especially with regard to lifestyle factors such as diet and physical activity. Furthermore, obesity treatment strategies should take these lifestyle factors into account in order to conquer the obesity epidemic.

Contributors S-AK: analysed the data and drafted, reviewed and edited the manuscript. SS: supervised the study. KL, J-KL and DK: reviewed and edited the manuscript and contributed to the discussion.

Funding This work was supported by the National Research Foundation of Korea (NRF) funded by the Korea government (MSIT) (NRF-2020R1C1C1014286).

\section{Competing interests None declared.}

Patient consent for publication Not required.

Ethics approval The study was approved by the Institutional Review Board (IRB) of Chung-Ang University, Anseong, Korea (IRB No. 0608-018-179).

Provenance and peer review Not commissioned; externally peer reviewed.

Data availability statement Data are available upon reasonable request. Data sharing of KoGES is available through the website of the Korea National Institute of Health (http://www.nih.go.kr/NIH).

Open access This is an open access article distributed in accordance with the Creative Commons Attribution Non Commercial (CC BY-NC 4.0) license, which permits others to distribute, remix, adapt, build upon this work non-commercially, and license their derivative works on different terms, provided the original work is properly cited, appropriate credit is given, any changes made indicated, and the use is non-commercial. See: http://creativecommons.org/licenses/by-nc/4.0/.

\section{ORCID iD}

Sangah Shin http://orcid.org/0000-0003-0094-1014

\section{REFERENCES}

1 Abarca-Gómez L, Abdeen ZA, Hamid ZA, et al. Worldwide trends in body-mass index, underweight, overweight, and obesity from 1975 to 2016: a pooled analysis of 2416 population-based measurement studies in 128.9 million children, adolescents, and adults. The Lancet 2017;390:2627-42.

2 Dai H, Alsalhe TA, Chalghaf N, et al. The global burden of disease attributable to high body mass index in 195 countries and territories, 1990-2017: an analysis of the global burden of disease study. PLoS Med 2020;17:e1003198.

3 Van Gaal LF, Mertens IL, De Block CE. Mechanisms linking obesity with cardiovascular disease. Nature 2006;444:875-80.

4 Lavie CJ, Milani RV, Ventura HO. Obesity and cardiovascular disease: risk factor, paradox, and impact of weight loss. J Am Coll Cardiol 2009;53:1925-32.
5 Stefan N, Birkenfeld AL, Schulze MB. Global pandemics interconnected - obesity, impaired metabolic health and COVID-19. Nat Rev Endocrinol 2021;17:135-49.

6 Frankenfield DC, Rowe WA, Cooney RN, et al. Limits of body mass index to detect obesity and predict body composition. Nutrition 2001;17:26-30.

7 Phillips CM. Metabolically healthy obesity: definitions, determinants and clinical implications. Rev Endocr Metab Disord 2013;14:219-27.

8 Stefan N, Häring H-U, Hu FB, et al. Metabolically healthy obesity: epidemiology, mechanisms, and clinical implications. Lancet Diabetes Endocrinol 2013;1:152-62.

9 Sims EA. Are there persons who are obese, but metabolically healthy? Metabolism 2001;50:1499-504.

10 Hamer M, Stamatakis E. Metabolically healthy obesity and risk of allcause and cardiovascular disease mortality. J Clin Endocrinol Metab 2012;97:2482-8.

11 Durward CM, Hartman TJ, Nickols-Richardson SM. All-cause mortality risk of metabolically healthy obese individuals in NHANES III. J Obes 2012;2012:460321.

12 Calori G, Lattuada G, Piemonti L, et al. Prevalence, metabolic features, and prognosis of metabolically healthy obese Italian individuals: the Cremona study. Diabetes Care 2011;34:210-5.

13 Hinnouho G-M, Czernichow S, Dugravot A, et al. Metabolically healthy obesity and risk of mortality: does the definition of metabolic health matter? Diabetes Care 2013;36:2294-300.

14 Zheng R, Zhou D, Zhu Y. The long-term prognosis of cardiovascular disease and all-cause mortality for metabolically healthy obesity: a systematic review and meta-analysis. J Epidemiol Community Health 2016;70:1024-31.

15 Eckel N, Meidtner K, Kalle-Uhlmann T, et al. Metabolically healthy obesity and cardiovascular events: a systematic review and metaanalysis. Eur J Prev Cardiol 2016;23:956-66.

16 Kramer CK, Zinman B, Retnakaran R. Are metabolically healthy overweight and obesity benign conditions?: a systematic review and meta-analysis. Ann Intern Med 2013;159:758-69.

17 International Obesity Task Force and World Health Organization. The Asian-Pacific perspective: redefining obesity and its treatment. Melbourne, Victoria, Australia: Health Communications Australia, 2000.

18 Cho YK, Kang YM, Yoo JH, et al. Implications of the dynamic nature of metabolic health status and obesity on risk of incident cardiovascular events and mortality: a nationwide population-based cohort study. Metabolism 2019;97:50-6.

19 Yang HK, Han K, Kwon H-S, et al. Obesity, metabolic health, and mortality in adults: a nationwide population-based study in Korea. Sci Rep 2016;6:1-10.

$20 \mathrm{Kim} \mathrm{NH}$, Seo JA, Cho H, et al. Risk of the development of diabetes and cardiovascular disease in metabolically healthy obese people: the Korean genome and epidemiology study. Medicine 2016;95:e3384.

21 Kim Y, Han B-G, KoGES group. Cohort profile: the Korean genome and epidemiology study (KoGES) Consortium. Int J Epidemiol 2017;46:e20.

22 Health Examinees Study Group. The health Examinees (HEXA) study: rationale, study design and baseline characteristics. Asian Pac J Cancer Prev 2015;16:1591-7.

23 Grundy SM, Cleeman JI, Daniels SR, et al. Diagnosis and management of the metabolic syndrome: an American heart Association/National heart, lung, and blood Institute scientific statement: Executive summary. Crit Pathw Cardiol 2005;4:198-203.

24 Schulze MB. Metabolic health in normal-weight and obese individuals. Diabetologia 2019;62:558-66.

25 World Health Organization. The 10th revision of the International classification of diseases. Available: https://www.who.int/ classifications/icd/icdonlineversions/en/ [Accessed 15 Jul 2020].

26 Aune D, Sen A, Prasad M, et al. BMI and all cause mortality: systematic review and non-linear dose-response meta-analysis of 230 cohort studies with 3.74 million deaths among 30.3 million participants. Bmj 2016;353.

27 Flegal KM, Kit BK, Orpana $\mathrm{H}$, et al. Association of all-cause mortality with overweight and obesity using standard body mass index categories: a systematic review and meta-analysis. JAMA 2013;309:71-82.

28 Soriguer F, Gutiérrez-Repiso C, Rubio-Martín E, et al. Metabolically healthy but obese, a matter of time? Findings from the prospective Pizarra study. J Clin Endocrinol Metab 2013;98:2318-25.

29 Bobbioni-Harsch E, Pataky Z, Makoundou V, et al. From metabolic normality to cardiometabolic risk factors in subjects with obesity. Obesity 2012;20:2063-9. 
30 Phillips CM, Perry IJ. Does inflammation determine metabolic health status in obese and nonobese adults? J Clin Endocrinol Metab 2013;98:E1610-9.

31 Muñoz-Garach A, Cornejo-Pareja I, Tinahones FJ. Does metabolically healthy obesity exist? Nutrients 2016;8:320.

32 Stefan N, Häring H-U, Schulze MB. Metabolically healthy obesity: the low-hanging fruit in obesity treatment? Lancet Diabetes Endocrinol 2018;6:249-58.

33 Arango-Lopera VE, Arroyo P, Gutiérrez-Robledo LM, et al. Mortality as an adverse outcome of sarcopenia. $J$ Nutr Health Aging 2013;17:259-62.

34 Stefan N. Causes, consequences, and treatment of metabolically unhealthy fat distribution. Lancet Diabetes Endocrinol 2020;8:616-27.

35 Stefan N, Schick F, Häring H-U. Causes, characteristics, and consequences of metabolically unhealthy normal weight in humans. Cell Metab 2017;26:292-300.

36 Jee SH, Kim MT, Lee SY, et al. Segregation analysis of waist circumference, hip circumference and waist-to-hip ratio in the Korean nationwide family study. Int J Obes Relat Metab Disord 2002;26:228-33.
37 Khang Y-H, Cho S-I, Kim H-R. Risks for cardiovascular disease, stroke, ischaemic heart disease, and diabetes mellitus associated with the metabolic syndrome using the new harmonised definition: findings from nationally representative longitudinal data from an Asian population. Atherosclerosis 2010;213:579-85.

38 Iso H, Sato S, Kitamura A, et al. Metabolic syndrome and the risk of ischemic heart disease and stroke among Japanese men and women. Stroke 2007;38:1744-51.

39 Chien K-L, Hsu H-C, Sung F-C, et al. Metabolic syndrome as a risk factor for coronary heart disease and stroke: an 11-year prospective cohort in Taiwan community. Atherosclerosis 2007;194:214-21.

40 Qiao Q, Laatikainen T, Zethelius B, et al. Comparison of definitions of metabolic syndrome in relation to the risk of developing stroke and coronary heart disease in Finnish and Swedish cohorts. Stroke 2009;40:337-43.

41 Sung K-C, Ryu S, Cheong ES, et al. All-cause and cardiovascular mortality among Koreans: effects of obesity and metabolic health. Am J Prev Med 2015;49:62-71.

42 Choi KM, Cho HJ, Choi HY, et al. Higher mortality in metabolically obese normal-weight people than in metabolically healthy obese subjects in elderly Koreans. Clin Endocrinol 2013;79:364-70. 